\title{
Modeling Individual and Group Actions in Meetings With Layered HMMs
}

\author{
Dong Zhang, Student Member, IEEE, Daniel Gatica-Perez, Member, IEEE, Samy Bengio, Member, IEEE, and \\ Iain McCowan, Member, IEEE
}

\begin{abstract}
We address the problem of recognizing sequences of human interaction patterns in meetings, with the goal of structuring them in semantic terms. The investigated patterns are inherently group-based (defined by the individual activities of meeting participants, and their interplay), and multimodal (as captured by cameras and microphones). By defining a proper set of individual actions, group actions can be modeled as a two-layer process, one that models basic individual activities from low-level audio-visual features, and another one that models the interactions. We propose a two-layer Hidden Markov Model (HMM) framework that implements such concept in a principled manner, and that has advantages over previous works. First, by decomposing the problem hierarchically, learning is performed on low-dimensional observation spaces, which results in simpler models. Second, our framework is easier to interpret, as both individual and group actions have a clear meaning, and thus easier to improve. Third, different HMM models can be used in each layer, to better reflect the nature of each subproblem. Our framework is general and extensible, and we illustrate it with a set of eight group actions, using a public five-hour meeting corpus. Experiments and comparison with a single-layer HMM baseline system show its validity.
\end{abstract}

Index Terms - Statistical models, multimodal processing and multimedia applications, human interaction recognition.

\section{INTRODUCTION}

Devising computational frameworks to automatically infer human behavior from sensors constitutes an open problem in many domains. Moving beyond the person-centered paradigm [36], recent work has started to explore multi-person scenarios, where not only individual but also group actions or interactions become relevant [11], [14], [31], [1].

One of these domains is meetings. The automatic analysis of meetings has recently attracted attention in a number of fields, including audio and speech processing, computer vision, human-computer interaction, and information retrieval [18], [38], [27], [3], [35], [4], [22]. Analyzing meetings poses a diversity of technical challenges, and opens doors to a number of relevant applications.

Group activity plays a key role in meetings [38], [27], and this is documented by a significant amount of work in social psychology [24]. Viewed as a whole, a group shares information, engages in discussions, and makes decisions, proceeding through diverse communication phases both in single meetings and during the course of a long-term teamwork [24]. Recognizing group actions is therefore useful for browsing and

Manuscript received June 7, 2004; revised Mar 9, 2005.

D. Zhang, D. Gatica-Perez, and S. Bengio are with IDIAP Research Institute, Martigny Switzerland. I. McCowan is with the eHealth Research Center Brisbane, Australia, and was with IDIAP when this work was completed. retrieval purposes [38], [22], e.g., to structure a meeting into a sequence of high-level items.

Interaction in meetings is inherently group-based [24] and multimodal [16]. In the first place, we can view a meeting as a continuous sequence of mutually exclusive group actions taken from an exhaustive set [22], [7]. Each of these group actions involves multiple simultaneous participants, and is thus implicitly constrained by the actions of the individuals. In the second place, as the principal modality in meetings, speech has recently been studied in the context of interaction modeling [13], [39], [7]. However, work analyzing the benefits of modeling individual and group actions using multiple modalities has been limited [1], [22], [23], [32], despite the fact that actions in meetings, both at the individual (e.g., note-taking or talking), and at the group level (e.g. dictating) are often defined by the joint occurrence of specific audio and visual patterns.

In this paper, we present a two-layer HMM framework for group action recognition in meetings. The fundamental idea is that, by defining an adequate set of individual actions, we can decompose the group action recognition problem into two levels, from individual to group actions. Both layers use ergodic HMMs or extensions. The goal of the lower layer is to recognize individual actions of participants using low-level audio-visual (AV) features. The output of this layer provides the input to the second layer, which models interactions. Individual actions naturally constitute the link between the low-level audio-visual features and high-level group actions. Similarly to continuous automatic speech recognition, we perform group action recognition directly on the data sequence, deriving the segmentation of group actions in the process. Our approach is general, extensible, and brings improvement over previous work, which reflects on the results obtained on a public meeting corpus, for a set of eight group actions based on multimodal turn-taking patterns.

The paper is organized as follows. Section II reviews related work. Section III introduces our approach. Section IV and Section V describe the meeting data and the feature extraction process respectively. Experiments and discussion are presented in Section VI. Conclusions are drawn in Section VII.

\section{RELATED WORK}

Current approaches to automatic activity recognition define models for specific activities that suit the goal in a particular domain, and use statistical methods for recognition. Predominately, the recognition of individual actions [36], or interaction 
involving few people [31], [14] has been investigated using visual features [15], [14], [31], [36], [40], although some work on the speech community can also be categorized as interaction recognition [13], [39]. In [13], recognition of a specific kind of interaction in meetings (agreement vs. disagreement) has been addressed using both word-based features (such as the total number of words, and the number of "positive" and "negative" keywords), as well as prosodic cues (such as pause, frequency and duration). In [39], the relationship between "hot spots" (defined in terms of participants highly involved in the discussion) and dialogue acts has been examined using contextual features (such as speaker identity or type of the meeting) and lexical features (such as utterance length and perplexity).

To our knowledge, however, little work has been conducted on recognition of group-based, multi-modal actions from multiple audio-visual streams captured by cameras and microphones [1], [22], [23]. [1] described automatic discovery of "influence" in a lounge room where people played interactive debating games. The so-called influence model, a Dynamic Bayes Network (DBN) which models group interactions as a group of Markov chains, each of which influences the others' state transitions, has been applied to determine how much influence each participant has on the others. Furthermore, our previous work presented different statistical sequence models to recognize turn-taking patterns in a formal meeting room scenario, where people discuss around a table and use a white-board and a projector screen [23], [22]. The analysis of multimodal group interactions has been explicitly addressed without distinguishing actions at individual and group levels.

Regarding statistical models, most of the existing work has used Hidden Markov Models (HMMs) [34] and extensions, including coupled HMMs, input-output HMMs, multi-stream HMMs, and asynchronous HMMs (see [29] for a recent review of models). Although the basic HMM, a discrete state-space model with an efficient learning algorithm, works well for temporally correlated sequential data, it is challenged by a large number of parameters, and the risk of overfitting when learned from limited data [30]. This situation might occur in the case of multimodal group action recognition where, in the simplest case, possibly large vectors of AV features from each participant are concatenated to define the observation space [22], [23].

The above problem is general, and has been addressed using hierarchical representations [41], [7], [30]. In [41], an approach for unsupervised discovery of multilevel video structures using hierarchical HMMs was proposed, in the context of sports videos. In this model, the higher-level structure elements usually correspond to semantic events, while the lower-level states represents variations occurring within the same event. In [7], two methods for meeting structuring from audio were presented, using multilevel DBNs. The first DBN model decomposed group actions in meetings as sequences of sub-actions, which have no explicit meaning and were obtained from training process. The second DBN model processed independently features of different nature, and integrate them at higher level. In both [41], [7], the lowlevel actions have no obvious interpretation, and the number of low-level actions is a model parameter learned during training, or set by hand, which makes the structure of the models difficult to interpret. The other work closest to ours is [30], in which layered HMMs were proposed to model multimodal office activities involving mainly one person at various time granularities. The lowest layer captured one video and two audio channels, plus keyboard and mouse activity features; the middle layer classifies $\mathrm{AV}$ features into basic events like "speech", "music", "one person", "nobody", etc. Finally, the highest layer uses the outputs of previous layers to recognize office activities with longer temporal extent. In this way, actions at different semantic levels and with different time granularities have been modeled with a cascade pyramid of HMMs. This hierarchical representation has been tested in SEER, a real-time system for recognizing typical office activities, and produced improvement over a simple baseline HMM.

The solution we present to the problem of group action recognition is novel. On one hand, unlike our previous work [22], [23], the framework presented here explicitly models actions at different semantic levels (from individual to group level) at the same time scale. This layered structure coincides with the structure of meetings as modeled in social psychology, that is, that meetings comprise individual actions and interactions [24]. On the other hand, our ultimate goal -modeling group activity- is different than that of [30]. Since the two HMM layers are trained independently, our framework is easy to interpret and enhanced at each of the levels. Unlike [30], we have studied a number of models suitable for multimodal data. For example, for the individual action layer, we use multistream HMMs [9] and asynchronous HMMs [2], which are more suitable to model multimodal asynchronous sequences. Furthermore, the type of sensors is also different. For our problem, the proposed work has a number of advantages, as described in the next section. A prelimary version of our work was first reported in [43].

\section{GROUP ACTION RECOGNITION}

In this section, we first introduce our computational framework. We then apply it to a specific set of individual and group actions. Finally, we describe some specific implementation details.

\section{A. Framework Overview}

Our framework is based on the use of Hidden Markov Models (HMMs) and some of their extensions. HMMs have been used with success for numerous sequence recognition tasks, including speech recognition [34]. HMMs introduce a hidden state variable and factorize the joint distribution of a sequence of observations and states using two simpler distributions, namely emission and transition distributions. Such factorization yields efficient training algorithms such as the Expectation-Maximization algorithm (EM) [5], which can be used to select the set of parameters to maximize the likelihood of several observation sequences. In our work, we use Gaussian Mixture Models (GMMs) to represent the emission distribution. 


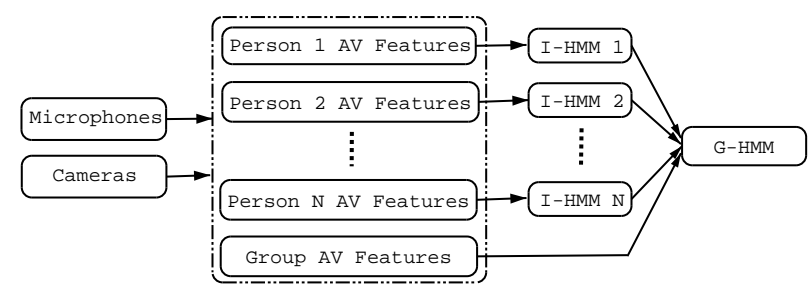

Fig. 1. Two-layer HMM framework

The success of HMMs applied to sequences of actions is based on a careful design of sub-models (distributions) corresponding to lexical units (phonemes, words, letters, actions). Given a training set of observation sequences representing meetings for which we know the corresponding labeling (but not necessarily the precise alignment), we create a new HMM for each sequence as the concatenation of sub-model HMMs corresponding to the sequence of actions. This new HMM can then be trained using EM and will have the effect of adapting each sub-model HMM accordingly. When a new sequence of observation features of a meeting becomes available, the objective is to obtain the optimal sequence of sub-model HMMs (representing actions) that could have generated the given observation sequence. An approximation of this can be done efficiently using the well-known Viterbi algorithm [37]. This process therefore leads to the recognition of actions directly on the data sequence, generating the action boundaries in the process.

In our framework, we distinguish group actions (which belong to the whole set of participants) from individual actions (belonging to specific persons). Our ultimate goal is the recognition of group activity, and so individual actions should act as the bridge between group actions and low-level features, thus decomposing the problem in stages. The definition of both action sets is thus clearly intertwined.

Let I-HMM denotes the lower recognition layer (individual action), and G-HMM denotes the upper layer (group action). I-HMM receives as input AV features extracted from each participant, and outputs recognition results, either as soft or hard decisions (Section III-C). In turn, G-HMM receives as input the output from I-HMM, and a set of group features, directly extracted from the raw streams, which are not associated to any particular individual. In our framework, each layer is trained independently, and can be substituted by any of the HMM variants that might capture better the characteristics of the data, more specifically asynchrony [2], or different noise conditions [9] between the audio and visual streams. Our approach is summarized in Figure 1. The training procedure is described in Section III-C.

Compared with a single-layer HMM, the layered approach has the following advantages, some of which were previously pointed out by [30]: (1) a single-layer HMM is defined on a possibly large observation space, which might face the problem of overfitting with limited training data. It is important to notice that the amount of training data becomes an issue in meetings where data labeling is not a cheap task. In contrast, the layers in our approach are defined over small-dimensional observation spaces, resulting in more stable performance in cases of limited amount of training data. (2) The I-HMMs are person-independent, and in practice can be trained with much more data from different persons, as each meeting provides multiple individual streams of training data. Better generalization performance can then be expected. (3) The GHMMs are less sensitive to slight changes in the low-level features because their observations are the outputs of the individual action recognizers, which are expected to be well trained. (4) The two layers are trained independently. Thus, we can explore different HMM combination systems. In particular, we can replace the baseline I-HMMs with models that are more suitable for multi-modal asynchronous data sequences, with the goal of gaining understanding of the nature of the data (Section III-C.1). The framework thus becomes simpler to understand, and amenable to improvements at each separate level. (5) The framework is general and extensible to recognize new group actions defined in the future.

\section{B. Definition of Actions}

As an implementation of the proposed framework, we define a set of group actions and individual actions in this section. On one hand, a set of group actions is defined based on multi-modal turn-taking patterns [23]. A solid body of work in social psychology has confirmed that, in the context of group discussions, speaker turn patterns convey a rich amount of information about the dynamics of the group and the individual behaviour of its members, including trends of influence, dominance, and interest [24], [33], [10]. While speaking turns are described mainly by audio information, significant information also exists in non-verbal cues. Work in the literature has studied how participants coordinate speaking turns via an ensemble of multimodal cues, such as gaze, speech back-channels, changes in posture, etc. [33], [21]. From a different perspective, recognizing multimodal group turntaking is also useful for meeting structuring, for access and retrieval purposes.

The list of group actions is defined in Table I. Note that we consider a "monologue" or a "presentation" as a group action, because we define it as the joint occurrence of several individual patterns (e.g., one person speaks while the others listen to her). For meeting browsing and indexing, it might be also desirable to know which specific participant is doing a monologue in the meeting. Therefore, we further divide the "monologue" action into "monologue1", "monologue2", etc., according to the number of participants. In a similar way, we divide the "monologue+note-taking" action into "monologue 1+note-taking", "monologue $2+$ note-taking", and so on. Thus, for a four-participant meeting, a set of $N_{G}=14$ group actions has been defined as: $N_{G}=\{$ discussion, monologue1, monologue1 + note-taking, monologue 2 , monologue 2 + note-taking, monologue 3 , monologue $3+$ note-taking, monologue 4 , monologue $4+$ note-taking, note-taking, presentation, presentation + note-taking, whiteboard, whiteboard + notetaking $\}$. These group actions are multimodal, and commonly found in meetings. For modeling purposes, they are assumed to define a partition (i.e., the action set is non-overlapping and exhaustive). This set is richer compared to the one that 
TABLE I

DESCRIPTION OF ACTIONS

\begin{tabular}{|c|c|}
\hline \multicolumn{2}{|r|}{ Group action description } \\
\hline Discussion & most participants engaged in a conversation \\
\hline Monologue & $\begin{array}{c}\text { one participant speaking } \\
\text { continuously without interruption }\end{array}$ \\
\hline $\begin{array}{l}\text { Monologue+ } \\
\text { Note-taking }\end{array}$ & $\begin{array}{l}\text { one participant speaking continuously } \\
\text { others taking notes }\end{array}$ \\
\hline Note-taking & most participants taking notes \\
\hline Presentation & $\begin{array}{l}\text { one participant presenting } \\
\text { using the projector screen }\end{array}$ \\
\hline $\begin{array}{l}\text { Presentation+ } \\
\text { Note-taking }\end{array}$ & $\begin{array}{l}\text { one participant presenting using } \\
\text { projector screen, others taking notes }\end{array}$ \\
\hline White-board & $\begin{array}{l}\text { one participant speaking } \\
\text { using the white-board }\end{array}$ \\
\hline $\begin{array}{l}\text { White-board+ } \\
\text { Note-taking }\end{array}$ & $\begin{array}{l}\text { one participant speaking using } \\
\text { white-board, others taking notes }\end{array}$ \\
\hline \multicolumn{2}{|r|}{ Individual action description } \\
\hline Speaking & one participant speaking \\
\hline Writing & one participant taking notes \\
\hline Idle & one participant neither speaking nor writi \\
\hline
\end{tabular}

we defined in [23], as it includes simultaneous occurrence of actions, like "monologue+note-taking" which could occur during real situations, like dictating or minute-taking. The group actions we defined here can be easily described by combinations of a proper set of individual actions defined in the following. Our framework is general, and other type of group actions could be defined. Note that high-level group actions in semantic terms (e.g. agreement / disagreement) would certainly require language-based features [13].

On the other hand, we define a small set of $N_{I}=3$ multimodal individual actions which, as stated earlier, will help bridge the gap between group actions and low-level AV features. The list appears in Table I. While the list of potentially interesting individual actions in meetings is large, our ultimate goal is recognition of the group-level actions. It is interesting to note that, although at first glance one would not think of "speaking" or "writing" as multimodal, joint sound and visual patterns do occur in these cases and are useful in recognition, as the results in later sections confirm.

Finally, meeting rooms can be equipped with white-boards or projector screens which are shared by the group. Extracting features from these group devices also helps recognize group actions. They constitute the group features described in the previous subsection. Their detailed description will be presented in section $\mathrm{V}$.

The logical relations between individual actions, group actions, and group features are summarized in Table II. The group actions can be seen as combinations of individual actions plus states of group devices. For example, "presentation + note-taking" can be decomposed into "speaking" by one individual, with more than one "writing" participant, while the group device of projector screen is in use. Needless to say, our approach is not rule-based, but Table II is useful to conceptually relate the two layers.

\section{Implementing the Two-layer Framework}

In this section, we present some details about the architecture of our framework. To facilitate description, we first define
TABLE II

RELATIONSHIPS BETWEEN GROUP ACTIONS, INDIVIDUAL ACTIONS AND GROUP FEATURES. THE SYMBOL " $\star$ " INDICATES THAT THE WHITE-BOARD OR PROJECTOR SCREEN ARE IN USE WHEN THE CORRESPONDING GROUP ACTION TAKES PLACE. SyMbOL "/" INDICATES THAT THE NUMBER OF PARTICIPANTS FOR THE CORRESPONDING ACTION IS NOT CERTAIN. THE NUMBERS $(0,1, \ldots)$ INDICATE THE NUMBER OF MEETING PARTICIPANTS INVOLVED IN THE GROUP ACTION.

\begin{tabular}{|c|c|c|c|c|c|}
\hline \multirow{2}{*}{ Group Actions } & \multicolumn{3}{|c|}{ Individual Actions } & \multicolumn{2}{|c|}{ Group Features } \\
\hline & speaki & writing & idle & white-boarc & d projector \\
\hline discussion & $>2$ & 1 & I & & \\
\hline monologue & 1 & 0 & I & & \\
\hline monologue+note-taking & 1 & $>=1$ & I & & \\
\hline note-taking & 0 & $>2$ & 0 & & \\
\hline presentation & 1 & 0 & I & & $\star$ \\
\hline presentation+note-taking & 1 & $>=1$ & I & & $\star$ \\
\hline white-board & 1 & 0 & I & $\star$ & \\
\hline white-board+note-taking & 1 & $>=1$ & I & $\star$ & \\
\hline
\end{tabular}

the following symbols:

- $\mathbf{O}^{a}$ : a sequence of audio-only feature vectors.

- $\mathbf{O}^{v}$ : a sequence of visual-only feature vectors.

- $\mathbf{O}^{a+v}$ : a sequence of concatenated audio-visual feature vectors.

- $\mathbf{o}_{1: t} \triangleq \mathbf{o}_{1}, \mathbf{o}_{2}, \ldots, \mathbf{o}_{t}$ : a sequence (audio, visual, or audiovisual stream) up to time $t$.

- $q_{t}$ : the HMM state at time $\mathrm{t}$

1) Individual Action Models: We investigate three models for the lower-layer I-HMM, each of which attempts to model specific properties of the data. For space reasons, the HMM models are described here briefly. Please refer to the original references for details [34], [9], [2]. The investigated models are:

Early Integration HMM (Early Int.), where a basic HMM [34] is trained on combined AV features. This method involves aligning and synchronizing the AV features to form one concatenated set of features which is then treated as a single stream of data. The concatenation simply defines the audiovisual feature space as the cartesian product of the audio and video feature spaces, creating vectors which first contain the components of the audio feature vector, followed by the components of the video feature vector. Early integration selects the set of parameters $\theta_{i}^{*}$ of the model corresponding to action $i$ that maximizes the likelihood of $L$ audio-visual observation sequences as follows:

$$
\theta_{i}^{*}=\arg \max _{\theta_{i}} \prod_{l=1}^{L} P\left(\mathbf{O}_{l}^{a+v} \mid \theta_{i}\right) .
$$

Audio-visual Multi-Stream HMM (MS-HMM), which combines the audio-only and visual-only streams. Each stream is modeled independently. $\theta_{i}^{*}=\left(\theta_{i, a}^{*}, \theta_{i, v}^{*}\right)$ are the best model parameters for action $i$ to maximize the likelihood of audioonly and visual-only sequences respectively,

$$
\theta_{i, a}^{*}=\arg \max _{\theta_{i, a}} \prod_{l=1}^{L} P\left(\mathbf{O}_{l}^{a} \mid \theta_{i, a}\right)
$$




$$
\theta_{i, v}^{*}=\arg \max _{\theta_{i, v}} \prod_{l=1}^{L} P\left(\mathbf{O}_{l}^{v} \mid \theta_{i, v}\right) .
$$

The final classification is based on the fusion of the outputs of both modalities by estimating their joint occurrence [9], as follows:

$$
P\left(\mathbf{O}_{l}^{a+v} \mid q_{t}\right)=P\left(\mathbf{O}_{l}^{a} \mid q_{t}, \theta_{i, a}\right)^{\omega} P\left(\mathbf{O}_{l}^{v} \mid q_{t}, \theta_{i, v}\right)^{(1-\omega)},
$$

where the weighting factor $\omega(0 \leq \omega \leq 1)$ represents the relative reliability of the two modalities.

Audio-visual Asynchronous HMM (A-HMM), which also combines the audio-only and visual-only streams, by learning the joint distribution of pairs of sequences when these sequences are not synchronized and are not of the same length or rate [2]. This situation could occur in the meeting scenario at the group level when, for instance, an individual starts playing her role before the rest of the group. A similar situation could happen at the individual level between the audio and visual streams. For instance, it is known that the movements of the face are not synchronized with the actually uttered speech of a person [20]. Furthermore, in a conversational setting, a person tends to move before taking a turn, and often stops gesticulating before finishing speaking as a turn-yielding signal [8]. Being able to stretch some streams with respect to others at specific points could thus yield performance improvement. The A-HMM for action $i$ models the joint distribution of the two streams by maximizing the likelihood of $L$ observation sequences as follows:

$$
\theta_{i}^{*}=\arg \max _{\theta_{i}} \prod_{l=1}^{L} P\left(\mathbf{O}_{l}^{a}, \mathbf{O}_{l}^{v} \mid \theta_{i}\right) .
$$

Furthermore, while normal HMM optimization techniques integrate the likelihood of the data over all possible values of the hidden variable (which is the value of the state at each time step), asynchronous HMMs also integrate this likelihood over all possible alignments between observation sequences, adding a new hidden variable $\tau_{t}=s$ meaning that observation $\mathbf{o}_{t}^{a}$ is aligned with observation $\mathbf{o}_{s}^{v}$. With the hidden variable $\tau_{t}$ and using several reasonable independence assumptions, the model in [2] can factor the joint likelihood of the data and the hidden variables into several simple conditional distributions, which makes the model tractable using the EM algorithm. The Viterbi algorithm can be used to obtain the optimal state sequence as well as the alignment between the two sequences.

2) Linking the Two Layers: Obviously, a mechanism to link the two HMM layers has to be specified. There are two approaches to do so, based on different I-HMM outputs. Let $a^{t}=\left(a_{1}^{t}, \ldots, a_{N_{I}}^{t}\right) \in \mathbb{R}^{N_{I}}$ denote a vector in a continuous space of dimension equal to the number of individual actions $\left(N_{I}\right)$, which indicates the degree of confidence in the recognition of each individual action at time $t$ for a sequence $\mathbf{o}_{1: t}$.

The first approach directly outputs the probability $P_{k}^{t}$ for each individual action model $M_{k}, k=1, \ldots, N_{I}$, as input feature vector to G-HMM, $a_{k}^{t}=P_{k}^{t}$ for all $k$. We refer to it as soft decision.

In soft decision, the probability $P_{k}^{t}$ of model $M_{k}$ given a sequence $\mathbf{o}_{1: t}$ is computed in two steps. In the first step, we compute the probability of having generated the sequence and being in the state $i$ at time $t$. We denote this probability as $\rho(i, t)$. For different I-HMMs, the probability $\rho(i, t)$ is computed in different ways.

\section{- Early integration normal HMM:}

$$
\rho(i, t)=\alpha(i, t),
$$

where $\alpha(i, t) \triangleq P\left(\mathbf{o}_{1: t}, q_{t}=i\right)$ is the forward variable in the standard Baum-Welch algorithm [34]. $\mathbf{o}_{1: t}$ could be audio-only, visual-only or audio-visual stream.

- Multi-stream HMM:

$$
\begin{aligned}
\rho(i, t) & =P\left(\mathbf{o}_{1: t}^{a}, \mathbf{o}_{1: t}^{v}, q_{t}=i\right) \\
& =P\left(\mathbf{o}_{1: t}^{a}, q_{t}=i\right)^{\omega} P\left(\mathbf{o}_{1: t}^{v}, q_{t}=i\right)^{1-\omega},
\end{aligned}
$$

where $\mathbf{o}_{1: t}^{a}$ is the audio-only sequence and $\mathbf{o}_{1: t}^{v}$ is the visual-only sequence. $\omega$ is the weighting factor defined in Equation (4).

- Asynchronous HMM:

$$
\rho(i, t)=\sum_{s=t-\triangle t}^{t+\triangle t} P\left(\mathbf{o}_{1: t}^{a}, \mathbf{o}_{1: s}^{v}, q_{t}=i, \tau_{t}=s\right),
$$

where $\Delta t$ is the size of a sliding window centered at current time $t$. The variable $\tau_{t}=s$ can be seen as the alignment between sequence $\mathbf{o}_{1: t}^{a}$ and $\mathbf{o}_{1: s}^{v}$.

In the second step, we normalize the probability $\rho(i, t)$ for all states of all the models. The probabilities of all states for all models sum up to one,

$$
\sum_{j=1}^{N_{S}} P\left(q_{t}=j\right)=1,
$$

where $N_{S}$ is the number of all states for all models. Then the probability $P\left(q_{t}=i \mid \mathbf{o}_{1: t}\right)$ of state $i$ given a sequence $\mathbf{o}_{1: t}$ is

$$
\begin{aligned}
P\left(q_{t}=i \mid \mathbf{o}_{1: t}\right) & =\frac{P\left(q_{t}=i, \mathbf{o}_{1: t}\right)}{P\left(\mathbf{o}_{1: t}\right)} \\
& =\frac{P\left(q_{t}=i, \mathbf{o}_{1: t}\right)}{\sum_{j=1}^{N_{S}} P\left(q_{t}=j, \mathbf{o}_{1: t}\right)} \\
& =\frac{\rho(i, t)}{\sum_{j=1}^{N_{S}} \rho(j, t)} .
\end{aligned}
$$

With this, the probability $P_{k}^{t}$ of model $M_{k}$ given a sequence $\mathbf{o}_{1: t}$ is then computed as

$$
\begin{aligned}
P_{k}^{t} & =\sum_{i \in M_{k}} P\left(q_{t}=i \mid \mathbf{o}_{1: t}\right) \\
& =\sum_{i \in M_{k}} \frac{\rho(i, t)}{\sum_{j=1}^{N_{S}} \rho(j, t)},
\end{aligned}
$$

where $i$ is the state in model $M_{k}$, which is a subset of the states of all models, and $N_{S}$ is the total number of states. The probability $P_{k}^{t}$ of model $M_{k}$ is the sum of the probabilities of all states in model $M_{k}$.

In the second approach, the individual action model with the highest probability outputs a value of 1 , while all other models output a zero value. The vector $a^{t}$ generated in this way is used as input to G-HMM. We refer to it as hard decision.

We concatenate the individual recognition vectors from all participants, together with the group-level features, into 


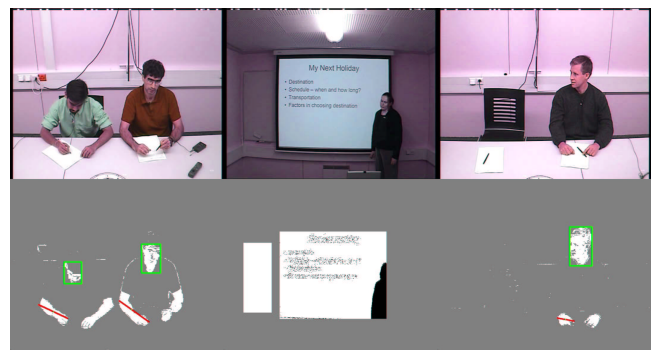

Fig. 2. Multi-camera meeting room and visual feature extraction

a $\left(N_{I} \times N_{P}+N_{G F}\right)$-dimensional vector (where $N_{P}$ is the number of participants, and $N_{G F}$ is the dimension of the group features) as observations to G-HMM for group action recognition.

\section{Meeting Data}

We used the publicly available meeting corpus we first described in [22], which was collected in a meeting room equipped with synchronized multi-channel audio and video recorders (publicly available at http://mmm.idiap.ch/). The sensors include three fixed cameras and twelve microphones [26]. Two cameras have an upper-body, frontal view of two participants including part of the table. A third wide-view camera captures the projector screen and white-board. Audio was recorded using lapel microphones for all participants, and an eight-microphone array placed in the center of the table. The complex nature of the audio-visual information present in meetings will be better appreciated by looking directly at the above website. A snapshot of the three camera views, and the visual feature extraction is shown in Figure 2. The corpus consists of 59 short meetings at five-minute average duration, with four participants per meeting. The group action structure was scripted before recording, so part of the group actions labels we define were already available as part of the corpus. However, we needed to relabel the rest of the group actions (e.g. monologues into either monologues or monologues+note-taking), and to label the entire corpus in terms of individual actions. All ground-truth was produced using Anvil, a publicly available video annotation tool (http://www.dfki.de/ kipp/anvil/).

\section{Multi-Modal Feature Extraction}

In this section, we describe the process to extract the two types of AV features: person-specific AV features and grouplevel AV features. The former are extracted from individual participants. The latter are extracted from the whiteboard and projector screen regions.

\section{A. Person-Specific AV Features}

Person-specific visual features were extracted from the cameras that have a close view of the participants. Person-specific audio features were extracted from the lapel microphones attached to each person, and from the microphone array. The complete set of features is listed in Table III.
Person-specific visual features. For each video frame, the raw image is converted to a skin-color likelihood image, using a 5-component skin-color Gaussian mixture model (GMM). We use the chromatic color space, known to be less variant to the skin color of different people [42]. The chromatic colors are defined by a normalization process: $r=\frac{R}{R+G+B}, g=$ $\frac{G}{R+G+B}$. Skin pixels were then classified based on thresholding of the skin likelihood. A morphological postprocessing step was performed to remove noise. The skin-color likelihood image is the input to a connected-component algorithm (flood filling) that extracts blobs. All blobs whose areas are smaller than a given threshold were removed. We use 2-D blob features to represent each participant in the meeting, assuming that the extracted blobs correspond to human faces and hands. First, we use a multi-view face detector to verify blobs corresponding to the face. The blob with the highest confidence output by the face detector is recognized as the face. Among the remaining blobs, the one that has the rightmost centroid horizontal position is identified as the right hand (we only extracted features from the right hands since the participants in the corpus are predominately right-handed). For each person, the detected face blob is represented by its vertical centroid position and eccentricity [36]. The hand blob is represented by its horizontal centroid position, eccentricity, and angle. Additionally, the motion magnitude for head and right hand are also extracted and summed into one single feature.

Person-specific audio features. Using the microphone array and the lapels, we extracted two types of person-specific audio features. On one hand, speech activity was estimated at four seated locations, from the microphone array waveforms. The seated locations are expressed as 3-D vectors in Cartesian coordinates, measured with respect to the microphone array in our meeting room. These vectors correspond to the location where people are typically seated. One measure was computed per seat location. The speech activity measure coming from each seated location was the SRP-PHAT (Steered Response Power-Phase Transform) measure, an increasingly popular technique used for acoustic source localization due to its suitability for reverberant environments [6]. SRP-PHAT is a continuous value that indicates the speech activity at a particular location. On the other hand, three acoustic features were estimated from each lapel waveform: energy, pitch and speaking rate. We computed these features on speech segments, setting a value of zero on silence segments. Speech segments were detected using the microphone array, because it is well suited for multiparty speech. We used the SIFT algorithm [19] to extract pitch, and a combination of estimators [28] to extract speaking rate.

\section{B. Group AV Features}

Group AV features were extracted from the white-board and projector screen regions. Given the constrained topology of a real meeting room, most people will naturally tend to occupy the same regions when making a presentation or using the whiteboard. The features are listed in Table III.

Group visual features. These were extracted from the camera that looks towards the white-board and projector screen 
TABLE III

AUdio-VISUAL FEATURE LIST

\begin{tabular}{|c|c|c|}
\hline \multirow{11}{*}{$\begin{array}{l}\text { Person- } \\
\text { Specific } \\
\text { Features }\end{array}$} & \multirow{5}{*}{ Audio } & Description \\
\hline & & SRP-PHAT from each seat \\
\hline & & speech relative pitch \\
\hline & & speech energy \\
\hline & & speech rate \\
\hline & \multirow{6}{*}{ Visual } & head vertical centroid \\
\hline & & head eccentricity \\
\hline & & right hand horizontal centroid \\
\hline & & right hand angle \\
\hline & & right hand eccentricity \\
\hline & & head and hand motion \\
\hline \multirow{4}{*}{$\begin{array}{c}\text { Group } \\
\text { Features }\end{array}$} & \multirow{2}{*}{ Audio } & SRP-PHAT from white-board \\
\hline & & SRP-PHAT from projector screen \\
\hline & \multirow{2}{*}{ Visual } & mean difference from white-board \\
\hline & & mean difference from projector screen \\
\hline
\end{tabular}

area. We first get difference images between a reference background image and the image at each time, in the white-board and projector screen regions (Figure 2). On these difference images, we use the average intensity over a grid of $16 \times 16$ blocks as features.

Group audio features. These are SRP-PHAT features extracted using the microphone array from two locations corresponding to the white-board and projector screen.

\section{EXPERIMENTS}

In this section, we first describe the measures used to evaluate our results, and then present results for both individual action recognition and group action recognition.

\section{A. Performance Measures}

We use the action error rate (AER) and the frame error rate (FER) as measures to evaluate the results of group action recognition and individual action recognition, respectively.

AER is equivalent to the word error rate widely used in speech recognition, and is defined as the sum of insertions (Ins: symbols that were not present in a ground truth sequence, but were decoded in the recognized sequence), deletions (Del: symbols that were present in a ground truth sequence, but were not decoded in the recognized sequence), and substitutions (Sub: symbols that were present in a ground truth sequence, but were decoded as a different symbol in the recognized sequence), divided by the total number of actions in the ground-truth, AER $=\frac{\text { Sub }+ \text { Del }+ \text { Ins }}{\text { total actions }} \times 100 \%$. For group action recognition, we have $N_{G}=14$ possible actions which in many cases have no clear-cut temporal boundaries. Furthermore, at least five actions occur in each meeting in the corpus. We believe that AER is a thus good measure to evaluate group action recognition, as we are more interested in the recognition of the correct action sequence rather than the precise time alignment of the recognized action segments.

However, AER overlooks the time alignment between recognized and target action segments. For individual action recognition, there are only $N_{I}=3$ possible actions. Furthermore, some streams (participants) in the corpus consist of only two individual actions (e.g., a person who talks

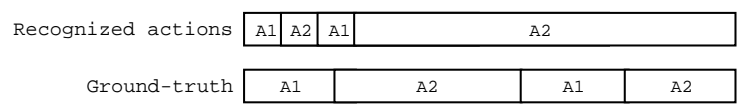

Fig. 3. AER is not a meaningful assessment for small number of actions.

only once during the course of a meeting). AER might not provide a meaningful assessment in such cases. As shown in Figure 3, AER equals zero because the recognized actions and the ground-truth actions have the same sequential order. But obviously, the result in Figure 3 is not perfect. Therefore, it is necessary to verify the temporal alignment of the recognized actions with another measure, especially for the case in which the total number of actions is small.

In this view, we adopt FER as the performance measure for individual action recognition. FER is defined as one minus the ratio between the number of correctly recognized frames and the number of total frames, FER $=\left(1-\frac{\text { correct frames }}{\text { total frames }}\right) \times$ $100 \%$. This measure reflects well the accuracy of the boundaries (begin and end time) of the recognized actions, compared to manually labeled action boundaries.

With limited number of training and testing actions, results are likely to vary due to the random initialization of the training procedure based on the EM algorithm [34]. For this reason, and to assess consistency in the results, we report the mean and standard deviation (STD) for AER and FER, computed over 10 runs with random initialization of the EM procedure.

Finally, we also use confusion matrices, whose rows and columns index the recognized and ground-truth actions, respectively. The element $c_{i j}$ of the confusion matrix corresponds to either the percentage (for individual actions) or the instances (for group actions) of action $j$ recognized as action $i$. The confusion matrix for group actions is based on AER, so there are substitution, insertion, and deletion errors. For individual actions, there are neither insertions nor deletions because the peformance measure is FER.

\section{B. Experimental Protocol}

For both individual and group action recognition, we use 6fold cross-validation on the training set to select the values of the model parameters that are not estimated as part of the EM algorithm. In a HMM/GMM architecture, these include the number of states per action, and the number of components (Gaussians) per state. In 6-fold cross-validation, we divided the data into 6 subsets of approximately equal size. We then train the models six times with different parameter configurations, each time leaving out one of the subsets from training, and using only the omitted subset to compute the corresponding performance measure (FER for individual actions, AER for group actions). The parameters resulting in the best overall performance were selected, and used to re-train the models on the whole training set.

For group actions, as described in [22], two disjoint sets of eight people each, whose identities were known, were used to construct the training and test sets. Each meeting was recorded using a randomly chosen 4-person combination within each of the sets. With this choice, no person appears in 
TABLE IV

NUMBER OF FRAMES $\left(N_{F}\right)$ AND NUMBER OF ACTIONS $\left(N_{A}\right)$ IN DIFFERENT DATA SETS

\begin{tabular}{|c|c|c|c|c|}
\hline \multirow{2}{*}{ Individual Actions } & \multicolumn{2}{|c|}{ train } & \multicolumn{2}{c|}{ test } \\
\cline { 2 - 5 } & $N_{F}$ & $N_{A}$ & $N_{F}$ & $N_{A}$ \\
\hline speaking & 35028 & 1088 & 33747 & 897 \\
writing & 15803 & 363 & 27365 & 390 \\
idle & 127569 & 1426 & 112488 & 1349 \\
total & 178400 & 2877 & 173600 & 2636 \\
\hline Group Actions & \multicolumn{2}{|c|}{ train } & \multicolumn{2}{c|}{ test } \\
\cline { 2 - 5 } & $N_{F}$ & $N_{A}$ & $N_{F}$ & $N_{A}$ \\
\hline discussion & 17760 & 48 & 14450 & 49 \\
monologue & 7615 & 26 & 7585 & 26 \\
monologue + note-taking & 6260 & 17 & 6695 & 23 \\
note-taking & 640 & 6 & 320 & 3 \\
presentation & 3170 & 6 & 3345 & 9 \\
presentation + note-taking & 3455 & 5 & 3865 & 9 \\
white-board & 2155 & 5 & 265 & 1 \\
white-board + note-taking & 3545 & 11 & 6875 & 19 \\
total & 44600 & 124 & 43400 & 139 \\
\hline
\end{tabular}

both the training and the test set. For individual actions, the original 8-people set in the training set was further split into two disjoint subsets at each time during the cross-validation procedure. One of these subsets was used to extract the streams belonging to the training set. The other subset was used to create the validation set. With this choice, we ensure that the data extracted from the same person is not used to both train and validate the individual action models.

From the 59 meetings, 30 are used as training data, and the remaining 29 are used for testing. The number of frames $\left(N_{F}\right)$ and number of actions $\left(N_{A}\right)$ for individual action and group action in the different data sets are summarized in Table IV. The number of individual actions is much larger than that of group actions. There are two reasons. First, for individual action recognition, there are four participants for each meeting. Therefore, there are $30 \times 4=120$ streams for training and $29 \times$ $4=116$ streams for testing. Second, the duration of individual actions is typically shorter than that of group actions.

\section{Individual Action Recognition}

The three methods described in Section III-C.1 were tested for individual action recognition.

Early integration (Early Int.), trained on three feature sets: audio-only, visual-only. and audio-visual.

Audio-visual multi-stream HMM (MS-HMM), combining individual audio and visual streams. Audio and visual streams are modeled independently. The final classification is based on the fusion of the outputs of both modalities by estimating their joint occurrence (Section III-C.1).

Audio-visual asynchronous HMM (A-HMM), combining individual audio and visual streams by learning the joint distribution of pairs of sequences when these sequences are not synchronized (Section III-C.1).

Multi-stream HMMs allow us to give different weights to different modalities 4 . Following the discussion presented in [23], we use $(0.8,0.2)$ to weight the audio and visual modalities, respectively. For asynchronous HMM, the allowed asynchrony ranges from $\pm 2.2 s$.
TABLE V

RESULTS OF INDIVIDUAL ACTION RECOGNITION

\begin{tabular}{|c|c|c|c|}
\hline Method & Features & FER (\%) & STD \\
\hline \multirow{3}{*}{ Early Int. } & Visual & 34.17 & 3.64 \\
\cline { 2 - 4 } & Audio & 23.48 & 2.70 \\
\cline { 2 - 4 } & Audio-visual & 9.98 & 2.65 \\
\hline MS-HMM & Audio-visual & 8.58 & 1.76 \\
\hline A-HMM & Audio-visual & 7.42 & 1.13 \\
\hline
\end{tabular}

TABLE VI

CONFUSION MATRIX OF RECOGNIZED INDIVIDUAL ACTIONS (USING VISUAL-ONLY FEATURES) ROWS: RECOGNIZED ACTIONS. COLUMNS: GROUND-TRUTH

\begin{tabular}{|c|c|c|c|}
\hline & Speaking & Writing & Idle \\
\hline Speaking & $51.92 \%$ & $3.00 \%$ & $8.22 \%$ \\
\hline Writing & $45.87 \%$ & $85.93 \%$ & $34.65 \%$ \\
\hline Idle & $2.21 \%$ & $11.07 \%$ & $57.13 \%$ \\
\hline
\end{tabular}

The summary of the results for all the individual action recognition models is presented in Table $\mathrm{V}$, in terms of FER mean and standard deviation, obtained over 10 runs (as described earlier, each run starts with a random initialization of the EM training procedure).

From Table V, we observe that all methods using AV features produced less than $10 \%$ FER, which is about $15 \%$ absolute improvement over using audio-only features, and about $25 \%$ absolute improvement over using visual-only features. Asynchronous HMM produced the best result. Given that the total number of frames is over 43,000 , the improvement using asynchronous HMM over the other HMM methods is statistically significant with a confidence level above $99 \%$, using a standard proportion test [12]. The improvement suggests that there exist asynchronous effects between the audio and visual modalities. Additionally, we tested the MS-HMM system with an equal-weight scheme $(0.5,0.5)$. The performance decreased compared to the MS-HMM with larger weight on audio $(0.8$ and 0.2 , see earlier discussion). This is not surprising given the predominant role of audio in the defined actions.

The confusion matrices for visual-only, audio-only, and audio-visual streams, corresponding to a randomly chosen single run, are shown in Tables VI, VII, and VIII, respectively. We can see that "speaking" is well detected using audio-only features, and that "writing" is well detected using visualonly features. Using audio-visual features, both "speaking" and "writing" are generally well detected. Using AV features, "writing" tends to get confused with "idle", which in turn is the action with the highest FER. This is likely due to the catch-all role that this action plays. In practice, "idle" includes all other possible AV patterns, (e.g. pointing, laughing, etc.), which makes its modeling more difficult, compared with the other two well-defined actions.

In order to empirically investigate asynchronous effects in the individual actions, we performed forced alignment decoding on the audio-only and visual-only streams independently. A similar approach was taken to establish empirical evidence for asynchrony in multi-band automatic speech recognition 
TABLE VII

CONFUSION MATRIX OF RECOGNIZED INDIVIDUAL ACTIONS (USING AUDIO-ONLY FEATURES) ROWS: RECOGNIZED ACTIONS. COLUMNS: GROUND-TRUTH

\begin{tabular}{|c|c|c|c|}
\hline & Speaking & Writing & Idle \\
\hline Speaking & $91.74 \%$ & $1.26 \%$ & $1.78 \%$ \\
\hline Writing & $1.16 \%$ & $35.23 \%$ & $22.10 \%$ \\
\hline Idle & $7.10 \%$ & $63.51 \%$ & $76.12 \%$ \\
\hline
\end{tabular}

TABLE VIII

CONFUSION MATRIX OF RECOGNIZED INDIVIDUAL ACTIONS (USING AV FEATURES) ROWS: RECOGNIZED ACTIONS. COLUMNS: GROUND-TRUTH

\begin{tabular}{|c|c|c|c|}
\hline & Speaking & Writing & Idle \\
\hline Speaking & $94.23 \%$ & $2.12 \%$ & $4.73 \%$ \\
\hline Writing & $1.03 \%$ & $89.60 \%$ & $10.89 \%$ \\
\hline Idle & $4.74 \%$ & $8.28 \%$ & $84.38 \%$ \\
\hline
\end{tabular}

in [25]. The decoder in each stream was constrained by the ground-truth individual action sequence, and so the output action sequences differ only in their temporal boundaries. We calculated the time misalignment (start-time difference of corresponding actions ) between the two sequences. Actions having absolute misalignments larger than $5 s$ were discarded, as the misalignments were more likely caused by recognition errors, rather than asynchronous effects. Figure 4 shows the resulting histogram of misalignments, assumed due to asynchronous effects, for these individual actions. The histogram can be approximated by a Gaussian distribution, with a mean of $-0.13 \mathrm{~s}$ (as misalignments happened in both directions) and a standard deviation of 2.05 . More than $80 \%$ of the individual actions are distributed in the range of $\pm 2.2 \mathrm{~s}$ (defined at the beginning of this section), while there are $17 \%$ individual actions without any asynchronous effects $(P(t=0)=17 \%)$. This suggests that, for most individual actions having evidence in both streams, allowing asynchrony between streams should model the data more accurately.

\section{Group Action Recognition}

Using the outputs from I-HMM and the group-level features, concatenated as described in Section III-C.2, we investigated a number of cases for recognition of group actions, as listed as follows.

1) Early integration, visual-only, soft decision. A normal HMM is trained using the combination of the results

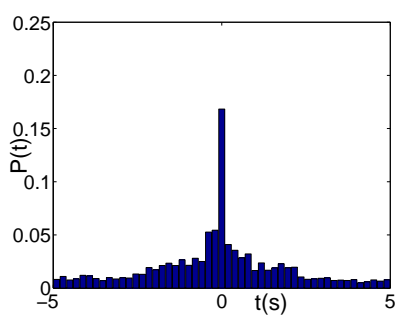

Fig. 4. Histogram of asynchronous effects of individual actions
TABLE IX

RESULTS OF GROUP ACTION RECOGNITION

\begin{tabular}{|c|c|c|c|c|}
\hline \multicolumn{2}{|c|}{ Method } & AER (\%) & STD \\
\hline \multirow{3}{*}{ Single-layer HMM } & \multicolumn{2}{|c|}{ Visual } & 48.20 & 3.78 \\
\cline { 2 - 5 } & \multicolumn{2}{|c|}{ Audio } & 36.70 & 4.12 \\
\cline { 2 - 5 } & \multicolumn{2}{|c|}{ Audio-visual } & 23.74 & 2.97 \\
\hline \multirow{3}{*}{ Two-layer HMM } & \multicolumn{2}{|c|}{ Visual } & 42.45 & 2.85 \\
\cline { 2 - 5 } & \multicolumn{2}{|c|}{ Audio } & 32.37 & 2.10 \\
\cline { 2 - 5 } & \multirow{2}{*}{ Early Int. } & hard & 17.98 & 2.75 \\
\cline { 2 - 5 } & & soft & 16.55 & 1.40 \\
\cline { 2 - 5 } & \multirow{2}{*}{ MS-HMM } & hard & 17.27 & 2.01 \\
\cline { 2 - 5 } & & soft & 15.83 & 1.61 \\
\cline { 2 - 5 } & \multirow{2}{*}{ A-HMM } & hard & 17.85 & 2.87 \\
\cline { 3 - 5 } & & soft & 15.11 & 1.48 \\
\hline
\end{tabular}

of the I-HMM trained on visual-only features, and the visual group features. The soft decision criteria is used.

2) Early integration, audio-only, soft decision. Same as above, but replacing visual-only by audio-only information.

3) Early integration, $A V$, hard decision. Same as above, but replacing visual-only by audio-visual information. The hard decision criteria is used.

4) Early integration, $A V$, soft decision. Same as above, but changing the criteria to link two HMM layers.

5) Multi-stream, $A V$, hard decision, using the multi-stream HMM approach as I-HMM. The hard decision criteria is used.

6) Multi-stream, $A V$, soft decision. Same as above, but changing the criteria to link two HMM layers.

7) Asynchronous HMM, AV, hard decision. We use the asynchronous HMM for individual action layer and audio-visual features. The hard decision criteria is used.

8) Asynchronous HMM, AV, soft decision. Same as above, but changing the criteria to link two HMM layers.

As baseline methods for comparison, we tested singlelayer HMMs, using low-level audio-only, visual-only, and AV features as observations [22], and trained by cross-validation following the same experimental protocol. The results appear in Table IX, in terms of AER mean and standard deviation over 10 runs. We observe from Table IX that the use of AV features outperformed the use of single modalities for both single-layer HMM and two-layer HMM methods. This result supports the hypothesis that the group actions we defined are inherently multimodal. Furthermore, the best two-layer HMM method (A-HMM) using AV features improved the performance by over $8 \%$ compared to the AV single-layer HMM. Given the small number of group actions in the corpus, a standard proportion test indicates that the difference in performance between AV single-layer and the best two-layer HMM is significant at the $96 \%$ confidence level. Additionally, the standard deviation for the two-layer approach is half the baseline's, which suggests that our approach might be more robust to variations in initialization, given the fact that each HMM stage in our approach is trained using an observation space of relatively low dimension. Regarding hard vs. soft decision, soft decision produced a slightly better result, although not statistically significant given the number of group actions. 
TABLE X

CONFUSION MATRIX OF RECOGNIZED GROUP ACTIONS FOR SINGLE-LAYER HMM USING AUDIO-VISUAL FEATURES. ROWS: RECOGNIZED ACTIONS. COLUMNS: GROUND-TRUTH

\begin{tabular}{|c|c|c|c|c|c|c|c|c|c|c|c|c|c|c|c|}
\hline & $\theta$ & $\bar{\Sigma}$ & $\begin{array}{l}\underline{\underline{t}} \\
\frac{+}{\Sigma}\end{array}$ & $\frac{\pi}{2}$ & 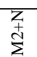 & $\sum$ & 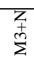 & $\frac{ \pm}{2}$ & $\begin{array}{l}\text { 丟 } \\
\end{array}$ & $z$ & a & 年 & 3 & $\frac{2}{3}$ & $\vec{\Xi}$ \\
\hline $\mathrm{D}$ & 45 & & & & & & & & & & & & & & 1 \\
\hline M1 & 2 & 6 & 3 & & & & & & & & & & & & \\
\hline $\mathrm{M} 1+\mathrm{N}$ & & & 3 & & & & & & & & & & & & \\
\hline M2 & & & & 6 & $\begin{array}{l}1 \\
3\end{array}$ & - & & & & & & & & & \\
\hline$\frac{\mathrm{M} 2+\mathrm{N}}{\mathrm{M}}$ & & & & 2 & & 2 & & 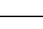 & & & 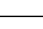 & & & & 1 \\
\hline $\mathrm{M} 3+\mathrm{N}$ & & & & & & 3 & 7 & & & & & & & & \\
\hline \begin{tabular}{|l|} 
M4 \\
\end{tabular} & & & & & & & & 2 & & & & & & & \\
\hline $\mathrm{M} 4+\mathrm{N}$ & & & & & & & & 3 & 5 & & & & & & \\
\hline $\begin{array}{ll}\mathrm{N} \\
\mathrm{P}\end{array}$ & & & & & & & & & & 2 & 6 & 5 & & & 1 \\
\hline \begin{tabular}{|c|}
$P+N$ \\
\end{tabular} & & & & & & & & & & & 1 & 3 & & & \\
\hline \begin{tabular}{|l|} 
W \\
\end{tabular} & 1 & & & & & 1 & & & & & 1 & & 1 & 2 & \\
\hline $\mathrm{W}+\mathrm{N}$ & & & & & & & & & & & & 1 & & 17 & \\
\hline In & & & & & 1 & & 1 & & & & & & & & \\
\hline
\end{tabular}

TABLE XI

CONFUSION MATRIX OF RECOGNIZED GROUP ACTIONS FOR TWO-LAYER HMM (USING ASYNCHRONOUS HMM WITH SOFT DECISION). ROWS: RECOGNIZED ACTIONS. COLUMNS: GROUND-TRUTH

\begin{tabular}{|c|c|c|c|c|c|c|c|c|c|c|c|c|c|c|c|}
\hline & 0 & $\bar{\Sigma}$ & $\frac{1}{\Sigma}$ & $\sum$ & $\stackrel{4}{\stackrel{4}{+}}$ & $\sum$ & $\begin{array}{l}4 \\
\frac{1}{2}\end{array}$ & $\frac{J}{2}$ & $\frac{\text { 奎 }}{2}$ & z & \& & 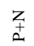 & 3 & $\begin{array}{l}Z \\
z \\
z\end{array}$ & $\overline{\check{g}}$ \\
\hline D & 44 & & & & & & & & & & & & & & \\
\hline M1 & 2 & 6 & 2 & & & & & & & & & & & & \\
\hline $\mathrm{M} 1+\mathrm{N}$ & & & 4 & & & & & & & & & & & & \\
\hline \begin{tabular}{|l|} 
M2 \\
\end{tabular} & & & & 7 & & & & & & & & & & & \\
\hline $\mathrm{M} 2+\mathrm{N}$ & & & & 1 & 5 & & & & & & & & & & \\
\hline \begin{tabular}{|l|} 
M3 \\
\end{tabular} & & & & & & 5 & & & & & & & & & 1 \\
\hline $\mathrm{M} 3+\mathrm{N}$ & & & & & & 1 & 6 & & & & & & & & \\
\hline \begin{tabular}{|l|} 
M4 \\
\end{tabular} & & & & & & & & 4 & & & & & & & \\
\hline $\mathrm{M} 4+\mathrm{N}$ & & & & & & & & 1 & 5 & & & & & & \\
\hline \begin{tabular}{|l|}
$\mathrm{N}$ \\
$\mathrm{D}$
\end{tabular} & & & & & & & 1 & & & 3 & & & & & \\
\hline \begin{tabular}{|c|}
$\mathrm{P}$ \\
\end{tabular} & & & & & & & & & & & 6 & & & & \\
\hline $\mathrm{P}+\mathrm{N}$ & & & & & & & & & & & & 8 & & & \\
\hline & 2 & & & & & & & & & & 2 & 1 & 1 & $\begin{array}{l}1 \\
18\end{array}$ & \\
\hline $\mathrm{W}+\mathrm{N}$ & & & & 1 & & & & & & & 1 & & & 18 & \\
\hline \begin{tabular}{|l|} 
Ins \\
\end{tabular} & & & & & & & & & & & & 1 & & & \\
\hline
\end{tabular}

However, the standard deviation using soft-decision is again around half the corresponding to hard-decision. Overall, the soft decision two-layer HMM appears to be favored by the results.

To further analyze results, we provide the confusion matrices for single-layer HMM using AV features, and two-layer HMM using AV, soft-decision and asynchronous HMM in Tables $\mathrm{X}$ and XI, respectively. We showed discussion (D), monologue (M1 ‥M4), monologue+note-taking $(\mathrm{M} 1+\mathrm{N} \cdots \mathrm{M} 4+\mathrm{N})$, note-taking $(\mathrm{N})$, presentation $(\mathrm{P})$, presentation+note-taking $(\mathrm{P}+\mathrm{N})$, white-board $(\mathrm{W})$, and white-board+note-taking $(\mathrm{W}+\mathrm{N})$. Empty cells represent zero values. It is evident that the twolayer method greatly reduced the number of errors, compared with the single-layer method. For both matrices, we see that most substitution errors come from confusions between actions with and without note-taking. This might be mainly because several instances of "writing" could not be reliably detected as individual actions, as mentioned in the previous subsection. There are several "presentation" actions confused with "white-board", which might be because some speakers moved around the white-board and projector-screen regions during a presentation. On the other hand, "discussion" and "note-taking” actions can be recognized reasonably well.
TABLE XII

RESULTS ON UNCONSTRAINED MEETINGS

\begin{tabular}{|c|c|c|}
\hline Method & $N_{R}$ & correct rate (\%) \\
\hline Single-layer HMM & 40 & 57.5 \\
\hline Two-layer HMM & 37 & 70.3 \\
\hline
\end{tabular}

\section{E. Recognizing Actions in Unconstrained Meetings}

To facilitate training and evaluation, the previous experiments were conducted on scripted meetings recorded in constrained conditions. To assess the proposed framework on natural multi-party conversations, we use a one-hour publicly available natural meeting recorded in the same setup, with which the AV single-layer HMM was compared to the best two-layer method, i.e., AV asynchronous HMM with softdecision. All parameters used for both methods are the same as in previous experiments.

The two methods were evaluated independently by two observers. The subjects watched and listened to the meeting recording, and judged the correctness of the actions automatically recognized using the single-layer and the two-layer methods. A final decision was made by the third person, for those actions in disagreement among each pair of observers. The results are shown in Table XII $\left(N_{R}\right.$ denotes the number of recognized actions for each system).

We can see that the results obtained with the two-layer HMM approach are better than those of the single-layer HMM, which again suggests the benefits of the proposed framework. For the one-hour natural meeting, over $70 \%$ group actions were correctly recognized using the layered method, which could be quite useful to meeting browsing and indexing. In practice, we noticed that it is difficult to determine clear-cut differences between the monologue and discussion actions, which constituted the main source of disagreement between the subjects that evaluated the results. Therefore, in future work, we need to address the ill-defined nature of some actions in real data.

\section{CONCLUSIONS AND FUTURE WORK}

In this paper, meetings were defined as sequences of multimodal group actions. We addressed the problem of modeling and recognizing such group actions, proposing a two-layer HMM framework to decompose the group action recognition problem into two layers. The first layer maps low-level AV features into individual actions. The second layer uses results from the first layer as input to recognize group actions. Experiments on a public 59-meeting corpus demonstrate the effectiveness of the proposed framework to recognize a set of multimodal turn-taking actions, compared to a baseline, single-layer HMM system. We believe our methodology to be promising. In the short term, we will explore its applicability to other sets of group actions, in multi-party conversations.

\section{ACKNOWLEDGMENTS}

This work was partly supported by the EU 5th FWP IST project M4 (IST-2001-34485), the EU 6th FWP IST Integrated 
Project AMI (FP6-506811, publication AMI-114), the EU 6th FWP IST PASCAL Network of Excellence (IST-2002506778), all three funded by the Swiss OFES, and by the Swiss NCCR IM2. We also thank Stephan Reiter (Technical University of Munich) for providing part of the groundtruth for individual actions, and Guillaume Lathoud (IDIAP Research Institute) for his work on audio feature extraction.

\section{REFERENCES}

[1] S. Basu, T. Choudhury, B. Clarkson, and A. Pentland. Towards measuring human interactions in conversational settings. In Proc. IEEE CVPR Workshop on Cues in Communication, Kawai, Dec. 2001.

[2] S. Bengio. An asynchronous hidden Markov model for audio-visual speech recognition. In S. Becker, S. Thrun, and K. Obermayer, editors, Proc. NIPS 15, 2003.

[3] P. Chiu, J. Boreczky, A. Girgensohn, and D. Kimber. LiteMinutes: An Internet-based system for multimedia meeting minutes. In Proc. Tenth World Wide Web Conference, 140-149, 2001.

[4] R. Cutler, Y. Rui, A. Gupta, J. Cadiz, I. Tashev, L. He, A. Colburn, Z. Zhang, Z. Liu, and S. Silverberg. Distributed meetings: A meeting capture and broadcasting system. In Proc. ACM Multimedia, 2002

[5] A.P. Dempster, N.M. Laird, and D.B. Rubin. Maximum Likelihood from Incomplete Data via the EM Algorithm. In Journal of Royal Statistical Society, vol. 39, no. 1, pp. 1-38, 1977.

[6] J. DiBiase, H. Silverman, and M. Brandstein. Robust localization in reverberant rooms. In M. Brandstein and D. Ward, editors, Microphone Arrays, chapter 8, pages 157-180. Springer, 2001.

[7] A. Dielmann and S. Renals. Dynamic Bayesian networks for meeting structuring. In Proc. IEEE ICASSP, 2004.

[8] S. Duncan. Some signals and rules for taking speaker turns in conversation. Journal of Personality and Social Psychology, vol. 23, no. 2, pp. 283-292, 1972.

[9] S. Dupont and J. Luettin. Audio-visual speech modeling for continuous speech recognition. IEEE Transactions on Multimedia, 2(3):141-151, Sep. 2000.

[10] N. Fay, S. Garrod, and J. Carletta. Group discussion as interactive dialogue or serial monologue: The influence of group size. Psychological Science, 11(6):487-492, 2000.

[11] A. Galata, N. Johnson, and D. Hogg. Learning behavior models of human activities. In British Machine Vision Conference, 1999.

[12] D. Gibbon, R. Moore, and R. Winksi. Handbook of Standards and Resources for Spoken Language Systems. Mouton de Gruyter, 1997.

[13] D. Hillard, M. Ostendorf, and E. Shriberg. Detection of agreement vs disagreement in meetings: Training with unlabeled data. In Proc. HLTNAACL Conference, Edmonton, May 2003.

[14] S. Hongeng and R. Nevatia. Multi-agent event recognition. In Proc IEEE ICCV, Vancouver, July 2001.

[15] D.M. Gavrila. The Visual Analysis of Human Movement: A Survey. Computer Vision and Image Understanding: CVIU, 73:82-98, 1999.

[16] R. Krauss and C. Garlock and P. Bricker and L. McMahon. The role of audible and visible back-channel responses in interpersonal communication. Journal of Personality and Social Psychology, 35(7):523529, 1977.

[17] G. Lathoud, I.A. McCowan. Location Based Speaker Segmentation. Proc. of ICASSP, 2003

[18] F. Kubala. Rough'n'Ready: a meeting recorder and browser. In $A C M$ Computing Surveys, 31, 1999.

[19] J. D. Markel. The SIFT algorithm for fundamental frequency estimation. IEEE Transactions on Audio and Electroacoustics, 20:367-377, 1972

[20] D. W. Massaro and D. G. Stork. Speech recognition and sensory integration. American Scientist, vol. 86, no. 3, pp. 236-244. 1998.

[21] D. Novick, B. Hansen, and K. Ward. Coordinating turn-taking with gaze. In Proc. Int. Conf. on Spoken Language Processing, 1996.

[22] I. McCowan, S. Bengio, D. Gatica-Perez, G. Lathoud, F. Monay, D. Moore, P. Wellner, and H. Bourlard. Modeling human interactions in meetings. In Proc. IEEE ICASSP, Hong Kong, April 2003.

[23] I. McCowan, D. Gatica-Perez, S. Bengio, G. Lathoud, M. Barnard, and D. Zhang. Automatic analysis of multimodal group actions in meetings. IEEE Transactions on Pattern Analysis and Machine Intelligence (PAMI), 27(3):305-317, 2005

[24] J. E. McGrath. Groups: Interaction and Performance. Prentice-Hall, 1984.
[25] N. Mirghafori and N. Morgan. Transmissions and Transitions: A Study of Two Common Assumptions in Multi-Band ASR. In Proc. ICASSP, Seattle, 1998

[26] D. Moore. The IDIAP smart meeting room. IDIAP-COM 07, IDIAP, 2002.

[27] N. Morgan, D. Baron, J. Edwards, D. Ellis, D. Gelbart, A. Janin, T. Pfau, E. Shriberg, and A. Stolcke. The meeting project at ICSI. In Proc. HLT Conference, San Diego, CA, March 2001

[28] N. Morgan and E. Fosler-Lussier. Combining multiple estimators of speaking rate. in Proc. ICASSP, 1998.

[29] K. Murphy. Dynamic Bayesian networks: Representation, inference and learning. Ph.D. dissertation, UC Berkeley, 2002.

[30] N. Oliver, E. Horvitz, and A. Garg. Layered representations for learning and inferring office activity from multiple sensory channels. In Proc. ICMI, October 2002.

[31] N. Oliver, B. Rosario, and A. Pentland. A Bayesian computer vision system for modeling human interactions. IEEE Transactions on Pattern Analysis and Machine Intelligence, 22(8), August 2000.

[32] E. Padilha and J. Carletta. Nonverbal behaviours improve a simulation of small group discussion. In Proc. First Int. Nordic Symposium of Multimodal Communication, Copenhagen, Sep. 2003.

[33] K. C. H. Parker. Speaking turns in small group interaction: A contextsensitive event sequence model. In Journal of Personality and Social Psychology, 54(6):965-971, 1988.

[34] L. R. Rabiner and B.-H. Juang. Fundamentals of Speech Recognition. Prentice-Hall, 1993.

[35] R. Stiefelhagen. Tracking focus of attention in meetings. IEEE International Conference on Multimodal Interfaces, Pittsburgh, PA, 2002.

[36] T. Starner and A. Pentland. Visual recognition of american sign language using HMMs. In Proc. Int. Work. on AFGR, Zurich, 1995.

[37] A.J. Viterbi. Error bounds for convolutional codes and an asymptotically optimal decoding algorithm. In IEEE Transactions on Information Processing, 13:260-269.

[38] A. Waibel, M. Bett, F. Metze, K. Ries, T. Schaaf, T. Schultz, H. Soltau, $\mathrm{H}$. Yu, and K. Zechner. Advances in automatic meeting record creation and access. in Proc. IEEE ICASSP, May 1999.

[39] B. Wrede and E. Shriberg. The relationship between dialogue acts and hot spots in meetings. In Proc. ASRU, Virgin Islands, Dec. 2003.

[40] C.R. Wren, A. Azarbayejani, T. Darrell and A. Pentland. Pfinder: Real-Time Tracking of the Human Body. IEEE Transactions on Pattern Analysis and Machine Intelligence, 19(7):780-785, 1997.

[41] L. Xie, S.-F. Chang, A. Divakaran, and H. Sun. Unsupervised discovery of multilevel statistical video structures using hierarchical hidden Markov models. in Proc. ICME, July 2003.

[42] J. Yang, L. Weier, and A. Waibel. Skin-color modeling and adaptation. in Proc. ACCV, 1998.

[43] D. Zhang, D. Gatica-Perez, S. Bengio, I. McCowan, and G. Lathoud Modeling Individual and Group Actions in Meetings: a Two-Layer HMM Framework. In Proc. IEEE CVPR, Workshop on Event Mining in Video (CVPR-EVENT), Washington DC, Jul. 2004.

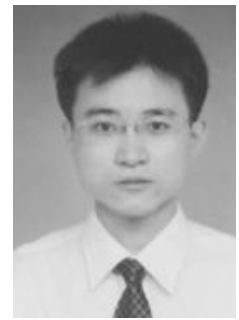

Dong Zhang obtained his B.E. (Automatic Control and Electrical Engineering) from Beijing Institute of Technology in 1998, and a M.S. (Computer Vision and Pattern Recognition) from the Institute of Automation, Chinese Academy of Sciences in 2001. Following this, he joined Microsoft Research Asia, where he did research and development in the area of multimedia retrieval. Since 2003 he has been a Ph.D. student at the IDIAP Research Institute in Switzerland. His research interests include machine learning, computer vision and multimedia systems. 


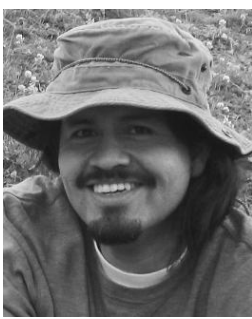

Daniel Gatica-Perez (S'01, M'02) received the B.S. degree in Electronic Engineering from the University of Puebla, Mexico in 1993, the M.S. degree in Electrical Engineering from the National University of Mexico in 1996, and the Ph.D. degree in Electrical Engineering from the University of Washington, Seattle, in 2001. He joined the IDIAP Research Institute in January 2002, where he is now a senior researcher. His interests include computer vision, multimodal signal processing, and multimedia information retrieval.

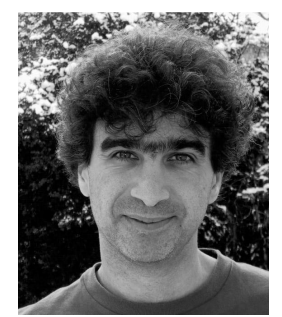

Samy Bengio (M'00, $\mathrm{PhD}$ in computer science, University of Montreal, 1993) is a senior researcher in statistical machine learning at IDIAP Research Institute since 1999, where he supervises Ph.D. students and postdoctoral fellows working on many areas of machine learning such as support vector machines, time series prediction, mixture models, largescale problems, speech recognition, multi channel and asynchronous sequence processing, multi-modal (face and voice) person authentication, brain computer interfaces, text mining, and many more. He is Associate Editor of the Journal of Computational Statistics, general chair of the Workshops on Machine Learning for Multimodal Interactions (MLMI'2004 and 2005) and was programme chair the IEEE Workshop on Neural Networks for Signal Processing (NNSP'2002). His current interests include all theoretical and applied aspects of learning algorithms.

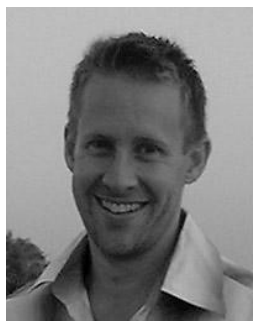

Iain McCowan (M'97) received the B.E. and B.InfoTech. from the Queensland University of Technology (QUT), Brisbane, in 1996. In 2001, he completed his $\mathrm{PhD}$ with the Research Concentration in Speech, Audio and Video Technology at QUT, including a period of research at France Telecom, Lannion. He joined the IDIAP Research Institute, Switzerland, in April 2001, as a Research Scientist, progressing to the post of Senior Researcher in 2003 While at IDIAP, he worked on a number of applied research projects in the areas of automatic speech recognition, content-based multimedia retrieval, multimodal event recognition and modeling of human interactions, in collaboration with a variety of academic and industrial partner sites. From January 2004, he was Scientific Coordinator of the EU AMI (Augmented Multi-party Interaction) project, a multi-disciplinary project involving researchers from 15 international sites, jointly managed by IDIAP and the University of Edinburgh. He joined the eHealth Research Centre, Brisbane, in May 2005 as Project Leader in Medical Imaging. 